\title{
Reduction in clinical assessment time using computer algorithms
}

\author{
JOSEPH P. LYONS and JOSEPH BROWN \\ Research Institute on Alcoholism, Buffalo, New York 14203
}

\begin{abstract}
A new mathematical procedure (BIDALGO) is suggested for application to the psychological testing field. BIDALGO, similar to past adaptive testing methods, is compared both with MMPI short-form efforts and applications in the educational testing literature. A comparison is also made between linear regression models used in past efforts and the Bayesian decision models used in BIDALGO. The requirements for an MMPI application are delineated, emphasizing branching procedures for specific scales and difficulties expected in achieving code-type congruence. Work in progress of applying BIDALGO to the depression scale and the full MMPI is also described.
\end{abstract}

Numerous objective psychological assessment instruments have been developed within the past few decades. In addition, aptitude, vocational interest, and clinical diagnostic instruments have evolved. These instruments are utilized both in research and in the direct assessment of patients in order to aid in the prescription of treatment programs. As the variety and number of instruments increases, the possibility of identifying the appropriate treatment modality for each type of client also increases. Consequently, research in developing instruments to identify unique patient types and classify treatment environments continues.

The most widely known and utilized of these objective instruments is the Minnesota Multiphasic Personality Inventory (MMPI) (Anastasi, 1976). Because of its renown, it will be used as an example within this article. It is important to note, however, that the procedures proposed throughout this paper are generalizable to the vast majority of objective assessment instruments.

\section{PROBLEM STATEMENT}

While the MMPI is the best known of the diagnostic instruments, it is not routinely utilized within the mental health field. One major reason is the time required to respond to the exceedingly large number of items composing the test. At present, a total of 566 item responses are collected with each MMPI administration. The average time needed for test administration is typically $1.5 \mathrm{~h}$ (Dahlstrom, Welsh, \& Dahlstrom, 1972). This time requirement limits the routine utilization of the MMPI in most mental health facilities. As noted by Kincannon (1968), "the occasion frequently occurs when a patient is unable or unwilling to complete either the individual or the group form of the MMPI .... There is also on occasion the need for rapid evaluation, such as for consultations" (p. 319).

This article attempts to briefly summarize some of the advances toward a solution to this problem. High- lighted is the contribution that computerization has already offered the psychological testing field, specifically the MMPI. A general model for shortening the MMPI is proposed and the necessary data structures and procedures are described. Finally, a specific application of the generalized model to alcoholism diagnosis is reported.

\section{BACKGROUND}

The impact of computer technology upon the psychological testing field is far too involved to outline here. Virtually all aspects of the testing field from the development stages of producing a new instrument to the administration, scoring, and interpretation of tests have in some way been affected by the use of computer technology.

Specific to the MMPI, much of the recent research attempting to define specialized scales from among the MMPI items have utilized complex statistical procedures available through standard commercial computer programs. Numerous scoring and interpretative services (Dahlstrom et al., 1972) are now offered to the clinician as an alternative to the tedious and less reliable method of hand scoring. Even the administration of the MMPI has been computerized (Lushene, O'Neil, \& Dunn, 1974).

Computer technology has also influenced the creation of short forms of the MMPI. As in the development of other instruments from the MMPI item pool, the utilization of automated complex statistical analyses has permitted a large number of short forms to be developed (Dean, 1972; Faschingbauer, 1973; Hugo, 1971; Kincannon, 1968; McLachlin, 1974; Overall \& GomezMont, 1974; Spera \& Robertson, Note 1). While reducing the administration time of the MMPI, these short forms have been faulted for a number of reasons (Johnson $\&$ Butcher, 1975). As aptly pointed out by Butcher and Tellegen (1978), "although MMPI short forms may correlate significantly with the full MMPI, the resulting 
code-type congruence (hit rate) between the two forms is quite low (from 33\% to $49 \%$ ), too low to result in very similar individual clinical decisions" (p.623).

MMPI code types can be succinctly defined as methods of reducing the MMPI profiles to more simplified and clinically meaningful groups. They are composed of the various possible configurations within the 13 scales. Numerous coding methods have been proposed throughout the years. While some have utilized detailed decision rules to codify any particular profile, others have simply relied upon the two or three most elevated scales. It is the latter methods, namely, the two- and three-point code types, that have been utilized most often in both research and clinical practice. Indeed, numerous "cookbooks" for interpretation of these code types have been published to aid the clinician in drawing relevant conclusions from the configural patterning of the MMPI scales. The vast majority of investigations attempting to compare short-form-originated code types with the full-form code types have reproduced the methods of Lichtenstein and Bryan (1966). Their method, designed to measure the stability of fullform MMPI profiles over relatively short test-retest intervals ( 1 or 2 days), included the computation of an overall Pearson product-moment correlation between the two test administrations. Furthermore, they determined the number of times that the highest scale on the first administration scored as the highest, second highest, third highest, or lower on the retest. Similarly, they determined the same for the second and third highest scales on the first administration as compared with their standing order at retest. Hit rates on 58 code types range from $36.7 \%$ (for the mini-mult) to $49.4 \%$ (for Faschingbauer's abbreviated MMPI). Analyses of these hit rates resulted in the discovery of excessive inter- and intra-short-form variability. Indeed, Butcher and Tellegen (1978) note that "the only MMPI short form recognized by the test authors and the publishers consists of a reduced number of items (around 400) that include all of the items required to score the 3 validity and 10 standard scales" (p. 623).

Up to this point, discussion has revolved about those short forms that have attempted to develop standardized paper-and-pencil instruments composed of a subset of the 566 items contained within the full-form MMPI. In contrast to this traditional testing methodology, several investigators have attempted to create "tailored" or "adaptive" testing methods (Lord, 1970; Weiss \& Betz, 1973). As described by Weiss (1979), "adaptive testing involves selecting for administration to a given individual the set of test items, from all the items available, that is likely to measure that individual best. This is in contrast to a conventional test in which everyone is administered the same items, as has been done in paperand-pencil tests almost since psychological testing began. In an adaptive test different people are administered different test items"'(p. 137).
Based primarily upon the latent trait theory first defined by Lord (1952), adaptive tests attempt to minimize the number of items administered while maximizing the precision of test results.

Adaptive test designs, utilizing the capabilities of computer logic and administration, have been shown to offer equal or greater test precision at significant savings in terms of time. Weiss (1979) reported on one particular study that compared adaptive achievement tests with conventional achievement tests. Achievement in biology as measured by an adaptive test was compared with achievement as measured by a conventional paper-andpencil test. The conventional test's length was 35 items, whereas the adaptive test's average length was only 27 items. Defining test precision in terms of information (which might also be expressed as the inverse of the standard error of measurement, a function of achievement level), the adaptive test proved more precise over all levels of achievement. Further reductions in the maximum number of items permitted for adaptive administration, although decreasing precision slightly relative to the unhindered adaptive test, still compared favorably with the precision of the conventional test. Thus, adaptive tests limited to no more than 20 items and exhibiting an average test length of 17 items performed equal to, or better than, the conventional achievement test in terms of precision. Weiss concluded that "these data show that adaptive tests can administer as few as $50 \%$ of the number of items in conventional tests and still provide measurements of higher quality" (1979, p. 145).

Clavelle and Butcher (1977), applying an adaptive testing methodology, attempted a typological approach to the vexing problem of code-type congruence. Suggesting that adaptive, on-line technology might aid in decreasing the number of items necessary for accurately typing any given test taken, they sought to accurately determine the two-point code types of a sample of 1,350 white adult psychiatric outpatients. Concentrating on only nine two-point code types, which collectively accounted for $53.5 \%$ of the sample MMPIs, Clavelle and Butcher delineated 69 items that best discriminated among pairs of code types. Utilizing the 10 most powerful items as an initial set, they attempted to type each subject's MMPIs into the nine code types. Based upon just this initial set, an average of $49 \%$ congruence was observed between the full-form code type and the adaptive code type. Successively increasing the number of items by 10 resulted in average congruence rates of $57 \%, 64 \%, 67 \%, 77 \%, 75 \%$, and $75 \%$ for $20,30,40,50$, 60 , and all 69 items, respectively. Developing probabilistic decision rules based upon the developmental sample, Clavelle and Butcher attempted to successively eliminate code types from consideration, so as to limit the number of possible code types that might be considered for a final typology.

This procedure tended to eliminate clusters of code 
types that were generally similar in terms of broader psychopathological labels. While Clavelle and Butcher's (1977) attempt was admittedly a preliminary excursion, it does suggest the viability of utilizing an adaptive approach to psychiatric screening in general and the MMPI in particular. They conclude: "It could well be that an adaptive approach to psychiatric screening (e.g., an abbreviated version of the MMPI) could result in the development of assessment procedures that are brief, efficient in their choice of items to be presented and clinically useful" (Clavelle \& Butcher, 1977, p. 859).

The remainder of this article proposes a general model that, when applied and implemented, will create a unique MMPI short-form for each individual test taker. This set of questions will then be used to calculate each of the scale scores and the most likely code type. It is expected that the code-type hit rates will exceed those presently reported by current short forms. Past efforts to reduce the time required to complete an MMPI type form relied upon statistical methods that identify common items for all clients. These methods are based on the assumption that both the control and clinical groups are representative of homogeneous populations. In truth, significantly different heterogeneous groups are presented in both control and clinical subpopulations. Given these assumptions, a system is needed that provides for the correct identification of the population group to which the client belongs and illuminates the idiosyncrasies of this member of the specified population group. Closely related to this issue is the effect of regression analyses in the development of many of the present short forms. Utilization of regression techniques for the estimation of full-scale scores from short-form response results in regression toward the mean, whereby "a particular individual will most likely be classified closer to the group mean on the variable being predicted that he did on the known variable" (Faschingbauer \& Newmark, 1978, p. 158).

A method that utilizes a common set of initial items to establish a foundation for inquiry and then selects the unique set of additional items to be reviewed for each individual client has proved to be a more effective identification tool in other diagnostic areas (Lyons \& Izadi, 1980) than one that establishes the set of items that can be used by all patients. It is expected that a variation in the method developed by Izadi (1979) will provide as consistent a hit rate on all MMPI scales as present short forms and will significantly improve the correct classification rate relevant to code-type specification.

\section{METHOD}

\section{Proposed Model for MMPI Item Reduction}

BIDALGO is an acronym for the Bijan Izadi diagnostic algorithm, a generalized mathematical model for diagnosis that utilizes three mathematical formulas to arrive at an initial diagnosis and a suggested set of additional questions to be asked of each client. The three formulas are: (1) a Bayesian diagnostic inference equation, (2) an information function, and (3) an implicit enumeration integer programming algorithm.

Bayesian diagnostic inference equation. Bayes' theorem is used to determine the probability that a client fits one of several diagnostic categories (populations) given he/she has certain symptoms (responses). Bayesian statistics have been applied to many health care diagnostic problems, such as heart (Bruce \& Yarnall 1966), liver (Burbank, 1966), thyroid (Overall \& Williams, 1963), and blood disorders (Lipkin, 1964). For illustrative purposes, a narrative description of Bayes' theorem applied to the problem of alcoholism symptoms of morning drinking, blackouts, and so on, is given here: Probability that a client is alcoholic, given he has morning drinking and blackouts, etc. $=$ (probability of alcoholism $X$ the probability of blackouts and morning drinking, etc., for a population of alcoholics/[(probability of alcoholism $X$ the probability of blackouts and morning drinking, etc., for a population of alcoholics) + (probability of nonalcoholism $X$ the probability of blackouts and morning drinking, etc., in nonalcoholics)] .

Mathematical computations (Barnoon \& Wolfe, 1972) become more involved as the number of symptoms increases and positive and negative responses are accounted for, but the conceptual nature of the problem formulation remains the same. Once these computations are completed for all symptoms under consideration, a complete diagnostic probability distribution, D, results. In the present system, the Bayesian formula is used to establish an initial diagnosis using a preestablished subset of the questions.

Information function. In addition to providing an initial diagnosis, the Bayesian equation is used as input into the information function. The potential informa. tion value of each possible additional question (Rs) can then be determined. The formula is as follows: $I(D / R)=$ $H(D)-H(D / R)$, where $I(D / R)$ refers to the information value in terms of bits of information that Question $R$ provides about the diagnostic probability distribution, $\mathrm{D}, \mathrm{H}(\mathrm{D})=$ the uncertainty of the Bayesian diagnosis about all categories' $D$, and $H(D / R)=$ the expected uncertainty of the Bayesian diagnosis given the additional Question $\mathrm{R}$ is examined.

The information gained about $\mathrm{D}$ by examining $\mathrm{R}$, $(D / R)$ can then be thought of as the subtraction of the uncertainty about $D$ given $R$ is examined [H(D/R)] from the initial uncertainty about $D$ given the initial Bayesian diagnosis [H(D)] .

Implicit enumeration function. Once the expected information value (in bits) has been calculated for each Question R, a third function, implicit enumeration (Wagner, 1969), is used to evaluate the gain in information and compare it with the clinical time required to complete the question.

The implicit enumeration determines the maximum information that can be attained within a specified 
time period. A helpful assumption to the formulation is that the information values remain in the same order of importance even if one of the symptoms is removed from consideration. Obviously, such an assumption is not valid when the initial Bayesian diagnosis is based on only a few questions. In simulation and clinical studies to date, the assumption has held when the diagnostic categories were well defined and the number of symptoms was greater than 15. However, examples representing all possible cases have not yet been tested. Izadi (1979) examines the problem in more depth than is possible here.

Final diagnosis. Once the clinician determines which questions will be asked and responses are solicited from the client, a final diagnosis can be determined by once again using the Bayesian diagnostic inference equation. However, for this final diagnosis, the Bayes equation will include responses from the initial question set plus those from the suggested set of additional questions that were suggested by BIDALGO and asked of the client. The calculations for the final diagnosis will be the same as for the initial diagnosis, but the total number of questions will have been expanded.

\section{Results in the Alcoholism Field}

The initial application of the BIDALGO was dubbed ADALGO (alcoholism diagnostic algorithm) and conducted on data collected at the Max Planck Institute of Psychiatry, in Germany (Ringer, Küfner, Antons, \& Feuerlein, 1977). The study involved the diagnosis of 120 alcoholics and 80 general hospital patients and compared the ADALGO system with a diagnostic criterion of 86 signs and symptoms and a procedure suggested by the National Council on Alcoholism (NCA) Criteria Committee (1972). The five-category system illustrated in Figure 1 was utilized. Diagnosis using two categories was also completed using both procedures. Table 1 summarizes the results of the application.

A second validation was completed on data from the Women's Health Survey (Russell \& Bigler, 1979), conducted in Buffalo, New York, during 1978-1979, which surveyed 2,627 obstetric and gynecology patients. All respondents completed a self-administered questionnaire. Medical record reviews and follow-up interviews were conducted for 233 patients. ADALGO and the diagnoses based on the NCA criteria were compared in this

Table 1

Comparison of the ADALGO Diagnostic Procedures With the NCA Procedure (Standard)

\begin{tabular}{cccc}
\hline & $\begin{array}{c}\text { Alcoholics } \\
(\mathrm{N}=120)\end{array}$ & $\begin{array}{c}\text { Nonalcoholics } \\
(\mathrm{N}=80)\end{array}$ & $\begin{array}{c}\text { Total } \\
(\mathrm{N}=200)\end{array}$ \\
\hline Five Categories & 100 & 68.75 & 87.5 \\
Two Categories & 100 & 85.00 & 94.0 \\
\hline
\end{tabular}

Note-The NCA classification for the two categories is based on the original rules suggested by the Criteria Committee. Values given are percentages.
Graphic Presentation of Five Category Diagnostic Schema and Verbal Equivalents

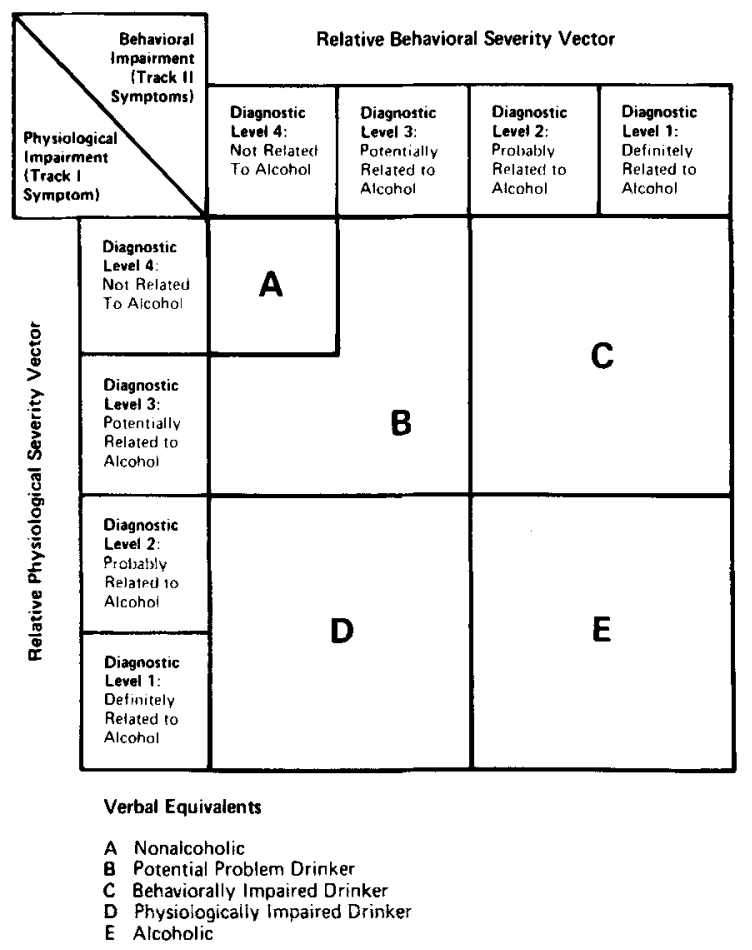

Figure 1.

second validation. The patients for which the selfadministered questionnaire, the medical record reviews, and the follow-up interviews were completed (233 patients) were classified according to the following categories: $69.5 \%$ nonalcoholic, $9.0 \%$ potential problem drinkers, $6.4 \%$ behaviorally impaired drinkers, $8.2 \%$ physiological impaired drinkers, and $6.9 \%$ alcoholics. The overall correction classification rate for five categories was $91 \%$ and was $95.7 \%$ for two categories.

In this second application, a limited amount of data relevant to women and their alcoholism symptoms was available to train ADALGO. Consequently, the initial ADALGO diagnoses were only $80 \%$ correct. A "prior-analysis" procedure was then developed through the use of a clinical logic-tree analysis based on the NCA criteria and procedure. The prior-analysis procedure was not used to determine which category was the most appropriate designation for the client, but it was used to logically eliminate diagnostic categories from further consideration. Using this new information, the computer was reprogrammed to adjust the prior probabilities within the Bayesian diagnostic inference equation and initiate the ADALGO process. The result increased the correct classification rate from $80 \%$ to $95.7 \%$ for the two categories. It is expected that as the data base for women expands, the correct classification rate for the ADALGO process coupled with prior analysis will approach $100 \%$ not only for two, but also for five diagnostic categories. 
A third application is presently being pilot tested in two health maintenance organizations (HMOs) in New York state. In each HMO, initial diagnostic data items are taken from the client's home history questionnaire. This questionnaire is completed by new subscribers upon enrollment and usually describes the enrollees' past medical history, problems, habits, and behaviors. Although most questionnaires do not ask alcoholism-related questions, a sufficient number of behavioral and physiological questions are asked that can be used to derive an initial diagnosis. Through the use of prior analysis along with the ADALGO system, the high-risk cases can be selected for further reveiw, which includes a medical record analysis for each client. Using the data gathered in the home history questionnaire and the medical record review, the high-risk cases can then be processed using ADALGO. At least three outcomes are possible: (1) The client is definitely not alcoholic and no further analyses are performed, (2) the client is definitely alcoholic and a list of his or her positive symptoms is printed and presented to the primary care staff member with a recommendation that alcoholism treatment be initiated, or (3) a probabilistic statement as the diagnosis of the client is printed along with the client's positive symptoms and a list of suggested tests and questions to be asked to confirm the diagnosis. In the case of this third outcome, the above information is printed on a colored sheet and entered into the client's record for review by the primary care clinician when the client returns for treatment.

Only those cases that have the highest probability of falling within an alcoholism category are singled out for further medical record review, and only those clients indicated by ADALGO are earmarked for further tests and questions. Consequently, a large HMO population can be screened for alcoholism and those who are identified as being at risk can be further diagnosed. This ADALGO-based screening and diagnosis system tends to keep agency costs to a minimum while also enabling the diagnosis and referral of those in need of treatment.

\section{BIDALGO Requirements for an MMPI Application}

It is suggested throughout this article that utilization of the computer's inherent abilities in the administration, scoring, and interpretation of psychological tests may result in the development of clinically efficient, reliable, and valid alternatives to the current assessment instruments. Specific to the MMPI, it is theorized that a BIDALGO application, using highly specified conditional probabilities within the Bayesian diagnostic inference equation, would result in scores similar to those recorded on the full MMPI form for each clinical scale. Furthermore, the issue of code-type congruence could be approached by one of the two following means: (1) Code types could be determined on the basis of the BIDALGO final diagnoses for each scale, or (2) code types could be determined on the basis of a subsequent separate BIDALGO application, specific to code-type delineation.

In either case, the end result would be an individually shortened MMPI set of items. Automated administration, scoring, and interpretation would further increase the potential for clinical utility, and inexpensive computer storage of data would facilitate both further research efforts and the development of localized norms (in the form of highly defined conditional probabilities).

BIDALGO has been computerized and validated on other populations. In order to apply it to the MMPI or other assessment instruments, the following criteria should be met: (1) Have a complete set of somewhat independent questions conditioned on each specific scale category. The scale categories and questions should be used in some clinical settings and should be acceptable to the majority of the professionals in that field. (2) Have a large data base that contains all of the questions and frequencies of all possible responses for each scale category according to Criterion 1 above. (3) Utilize a precise diagnostic procedure developed by and acceptable to the majority of all professionals in that field as a standard of comparison (e.g., full-form MMPI). (4) Have a set of mutually exclusive and collectively exhaustive diagnostic categories related to each scale or disease under consideration. (5) Determine from clinical opinion the prior probability of each new client being in each of the scale categories according to the clinical or research setting in which BIDALGO will be applied.

The authors are in the process of applying BIDALGO to the MMPI, starting with the depression scale. The first application strategy to be attempted will be to separate each scale into score categories based upon the raw-score means and standard deviations of the Minnesota normative groups.

BIDALGO will then be utilized to classify the client's responses into one of the categories. Once the scales are calculated, the two most elevated categories over all scales will be designated as the code type. If some scales are typed for highest elevation, a specialized application of BIDALGO will be initiated using conditional probabilities keyed on raw scores along with the responses to the set of questions suggested in the first application of BIDALGO. In some cases, it is expected that the client will be asked a few additional questions in order to further pinpoint the code type.

\section{DISCUSSION}

The BIDALGO system is expected to be generalizable to numerous current assessment instruments. It has been shown to be applicable to alcoholics, as well as normal populations. The end result of a successful application of BIDALGO to various tests would be the increased utilization of standardized, objective instru- 
ments in the mental health and human services fields.

Since the use of computers to administer, score, and interpret assessment instruments has been expanded at an increased rate, one can expect more computerization in the future. If the system proposed in this paper proves effective, the computer will be used to develop assessment instruments and procedures that would not be possible without it. As stated by Johnson and Giannetti (1979), "on-line technology allows the use of the computer's decision capabilities, computing power, and data banks throughout the testing process" (p. 76). In essence, if the BIDALGO application proves successful, logic and quantitative capabilities will be programmed into the computer and combined with its speed and reliability to produce more suitable diagnostic and research tools.

\section{REFERENCE NOTE}

1. Spera, J., \& Robertson, M. A 104-item MMPI the maxi-mult. Paper presented at the Annual Meeting of the American Psychological Association, New Orleans, 1974.

\section{REFERENCES}

Anastasi, A. Psychological testing (4th ed.). New York: Macmillan, 1976.

Barnoon, S., \& Wolfe, H. Measuring the effectiveness of medical decisions. Springfield, Ill: Thomas, 1972.

Bruce, R. A., \& Yarnall, S. R. Computer-aided diagnosis of cardiovascular disorders. Journal of Chronic Diseases, 1966, $19,473-484$.

Burbank, F. A computer diagnostic system for the diagnosis of prolonged undifferentiating disease. American Journal of Studies on Alcohol, 1966, 27, 648-668.

Butcher, J. N., \& Tellegren, A. Common methodological problems in MMPI research. Journal of Consulting and Clinical Psychology, 1978, 46, 620-628.

Clavelle, P. R., \& Butcher, J. N. An adaptive typological approach to psychiatric screening. Journal of Consulting and Clinical Psychology, 1977, 45, 851-859.

Dahlstrom, W. G., Welsh, S. G., \& Dahlstrom, L. E. $A n$ MMPI handbook (Vol. 1): Clinical interpretation. Minneapolis: University of Minnesota Press, 1972.

DEAn, E. F. A lengthened mini: The midi-mult. Journal of Clinical Psychology, 1972, 28, 68-71.

Faschingbauer, T. R. A short written form of the group MMPI (Doctoral dissertation, University of North Carolina, 1977). Dissertation Abstracts International, 1973, 34, 409B.

Hoffman, N. G., \& Butcher, J. N. Clinical limitations of three MMPI short forms. Journal of Consulting Clinical Psychology, 1975, 43, 32-39.

Hugo, J. Abbreviation of the Minnesota Multiphasic Personality
Inventory through multiple regression (Doctoral dissertation, University of Alabama, 1971). Dissertation Abstracts International, 1971, 32, 123B.

IzADI, B. M. Algorithms for aiding the clinician in medical diagnosis and referral: An application to alcoholism. Unpublished doctoral dissertation, State University of New York at Buffalo, 1979.

Johnson, J. H., \& Giannetti, R. A. Psychological systems questionnaire: A decision-relevant patient screening test designed for on-line administration. Journal of Medical Systems, 1979, 3, 75-80.

Kincannon, J. D. Prediction of the standard MMPI scale sources from 71 items: The mini-mult. Journal of Consulting and Clinical Psychology, 1968, 32, 319-325.

Lichtenstein, E., \& Bryan, J. H. Short-term stability of MMPI profiles. Journal of Consulting Psychology, 1966, 30, 172-174.

LIPKIN, M. The likelihood concept in differential diagnosis. Perspectives in Biology and Medicine, 1964, 7, 485-497.

LoRD, F. M. A theory of test scores. Psychometric Monograph, $1952,7,1-84$.

LoRD, F. M. Some test theory for tailored testing. In W. H. Holtzman (Ed.), Computer assisted instruction, testing and guidance. New York: Harper \& Row, 1970.

Lushene, R. D., O'Neil, H. F., \& DunN, T. Equivalent validity of a completely computerized MMPI. Journal of Personality Assessment, 1974, 38, 353-361.

Lyons, J. P., \& Izadi, B. M. Computer-aided diagnosis of alcoholism. Journal of Studies on Alcohol, 1980, 41, 448-455.

McLachlin, J. F. C. Test-retest stability of long and short form MMPI scales over two years. Journal of Clinical Psychology, $1974,30,189-191$.

National Council on Alcoholism Criteria Committee. Criteria for the diagnosis of alcoholism. American Journal of Psychiatry, 1972, 129, 127-135.

Overall, J. E., \& Gomez-Mont, F. The MMPI 168 for psychiatric screening. Educational and Psychological Measurement, 1974, 34, 315-319.

Overall, J. E., \& Williams, C. M. Conditional probability program for diagnosis of thyroid function. Journal of the American Medical Association, 1963, 183, 307-313.

Ringer, C., Kufner, H., Antons, R., \& Feuerlein, W. The NCA Criteria for the diagnosis of alcoholism: An empirical evaluation study. Journal of Studies on Alcohol, 1977, 38, 1259-1273.

Rus8ell, M., \& Biglen, L. Screening for alcohol-related problems in an outpatient obstetric-gynecologic clinic. American Journal of Obstetrics and Gynecology, 1979, 134, 4-16.

Wagner, H. M. Principles of operations research. Englewood Cliffs, N.J: Prentice-Hall, 1969.

WEIss, D. J. Computerized adaptive achievement testing. In H. F. O'Neil (Ed.), Procedures for instructional systems development. New York: Academic Press, 1979.

WE1ss, D. J., \& BETZ, N. E. Ability measurement: Conventional or adaptive? (Research Report 73-1). Minneapolis: University of Minnesota, Department of Psychology, Psychometric Methods Program, 1973. (NTIS No. AD-757788). 